\title{
LA QUESTION DE L'ANTIQUITÉ DE L'HOMME AMÉRICAIN
}

\author{
Par H. VIGNAUd \\ Président de la Société des Américanistes de Paris.
}

La question de l'antiquité de l'homme américain est une de celles qui ont le plus occupé le Bureau d'Ethnologie à Washington, où rien n’a été négligé pour l'approfondir et pour la faire connaitre sous sa véritable face. Dans ce but, cette belle institution, a laquelle on doit de si nombreux et si remarquables travaux, a institué, à différentes reprises, des enquêtes approfondies et quelquefois des excursions scientifiques qui, sous la direction de savants autorisés, ont visité les localités où l'on avait constaté l'existence de squelettes, de crânes ou de débris osseux attribués à l'homme primitif, et ont étudié avec soin, non seulement ces restes ostéologiques, mais les terrains où ils avaient été recueillis et les conditions dans lesquelles ces trouvailles avaient été faites.

Ces enquétes se classent, naturellement, en deux grandes catégories selon quil s'agit d'ossements trouvés dans l'une ou l'autre des deux Amériques. Le premier des deux volumes dont on donne ici les titres ${ }^{1}$ ne se rapporte qu'à l'Amérique du Nord. C'est un rapport rédigé par Aleš Hrdlička, l'un des anthropologistes les plus compétents du Bureau d'Ethnologie et, certainement, celui de ses membres qui met le plus d'application à ce genre d'études.

Dans une courte introduction, ce savant rappelle les précautions que l'on doit prendre dans les fouilles qui ont pour objet la recherche de restes ostéologiques anciens; il explique les nombreuses causes d'erreurs qu'elles

1. Aleś Hrduččk. Skeletal remains suggesting or altributed to early man in North America. (Restes squelettiques attribués à l'homme primitif dans l'Amérique du Nord ou suggérant son existence.) Bureau of American Éthnology. Bulletin $n^{\circ} 33$. Washington, $1907,8^{\circ}$, pp. 113, pl.

-. Early man in South America (L'homme primitif dans l'Amérique du Sud.) En collaboration avec W. H. Holmes, Bailley Willis, Fred. Eug. Wright, et Clarence N. Fenner. Bureau of American Ethnology. Bulletin no 62. Washington, 1912, 8, pp. 405, pl. 